\title{
Huge cosmic lenses
}

Penny D. Sackett

Kapteyn Astronomical Institute, Groningen, The Netherlands

Cosmic lenses, islands of concentrated mass drifting in the $\checkmark$ vast void of space, span approximately a factor of $10^{20}$ in mass and $10^{16}$ in linear size. This enormous diversity, the longrange nature of the gravitational force, and the simplicity of the underlying physics, make gravitational lensing a powerful tool with which to study a wide variety of astronomical problems.

\section{The Phenomenon of Gravitational Lensing}

Astronomical theories are tested primarily through passive observation of the multi-coloured electromagnetic radiation that chances to intercept Earth's orbit on its journey from a distant energetic source. When this radiation passes near a mass concentration, its path is altered by the gravitation field associated with the over-density. The light is bent toward the mass, the effect of an enormous converging lens.

In principle, the bending is gradual and continuous, but because the Universe is so sparse, the thin lens approximation of optics is applicable: the primary effect is a (relatively) sudden bending through an angle $\alpha$ that depends on the size of the impact parameter $\xi$ compared to the Schwarzschild radius $R_{s}$ of the lens

$$
\alpha=2 R_{s} / \xi=(4 G M) /\left(c^{2} \xi\right),
$$

where $M$ is the lens mass (Fig. 1). If the lens is extended over distances comparable to or larger than the impact parameter, the total effect is computed by integrating over the density distribution of the lens.

The ray-tracing process can be expressed as the two-dimensional Jacobian matrix $J$ that describes how positions on the emitting source are mapped onto positions in the image plane. The symmetric piece of the diagonal elements of $J$ is related to the convergence of the lens (isotropic magnification of the images), while the anti-symmetric portion is related to the lens shear (shape of the images). The magnification at any position is given by the inverse of the matrix determinant. The loci of positions in the source plane at which this magnification is formally infinite (because $|\mathrm{J}|=0$ ), are called caustics; the corresponding image positions lie on critical curves.

A simple point lens has a point caustic at the angular position of the lens. Due to symmetry, a source located directly behind a point lens will be imaged at very large magnification (finite only due to the non-zero size of the source) into a circular critical curve centred on the lens position. The size of this circle is given by the angular Einstein radius

$$
\theta_{\mathrm{E}}=\sqrt{ }\left[\left(4 \mathrm{GM} D_{L S}\right) /\left(\mathrm{c}^{2} D_{O L} D_{O S}\right)\right],
$$

where $D_{L S}, D_{O L}$ and $D_{O S}$ are line-of-sight distances between the lens, source, and observer. Complicated, multiple and disjoint caustic and critical curves can be generated by compound or extended lenses (see, e.g., Fig. 2).

\section{Geometry of the Universe}

Lensing was first observed early in the last century, when a solar eclipse allowed the apparent positions of background stars near 


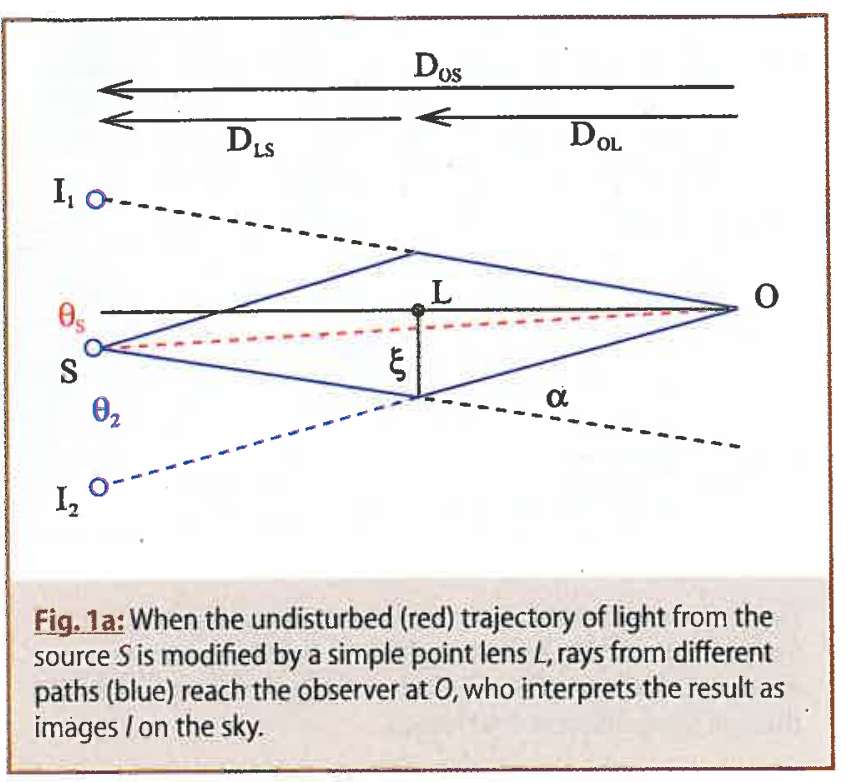

the limb of the Sun to be compared to their relative positions six months later when the (lensing) Sun was no longer in the line of sight. Some researchers anticipated the impact of this phenomenon on astronomy early on, but the potential began to be realized only in the two decades after the first observation of extragalactic lensing - multiple images of distant quasars - by radio astronomers in 1979.

Quasars are compact, extremely energetic, and often variable sources of emission. If lensed by an intervening massive galaxy, multiple images of a quasar can be formed, separated by about an arc second (see Eq. 2). Light associated with each image takes a different path through the lensing potential, resulting in geometric and potential time delays that, for variable quasars, can be measured by correlating the temporal light curves of the images. The total time delay is inversely proportional to Hubble's constant, $H_{0}$, the current expansion rate of the Universe. In this way, several lensed quasars with measured lens and source red shifts, and reasonably constrained lens mass models, have been used to infer $H_{0}$, result- ing in consistent, though often somewhat lower values than those relying on so-called "standard astronomical candles."

The expansion rate changes over cosmic time in a manner that depends on the matter density of the Universe and the value of the cosmological constant, $\Lambda$. Since the lensing probability at a given time depends on the volume per unit red shift, it is dependent on $\Lambda$. In the early 1990 s, several workers used the

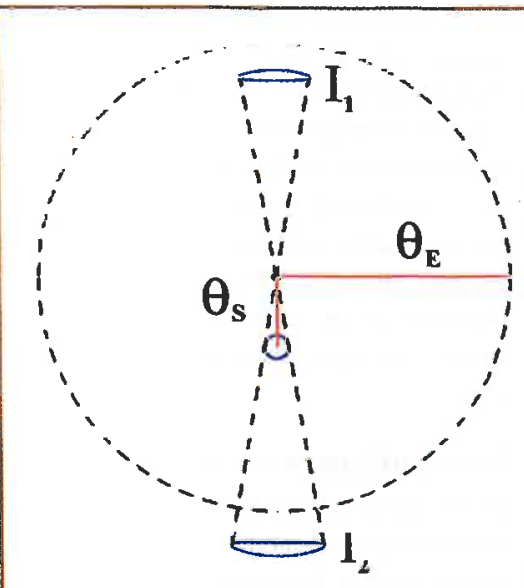

Fig. 1b: On the sky plane, these images are distorted into arclets, with angular separations similar to or greater than $2 \theta_{E}$. observed lensing rate as a function of red shift to derive the limit $\Lambda \leq 0.7$, consistent with the value of $\Lambda \approx 0.7$ now inferred from distant supernovae and microwave background studies.

\section{Harnessing the Power of Nature's Telescopes}

Focusing celestial radiation into sharp images and detailed spectra has been the work of skilled lens and instrument makers for several centuries. Only recently have astronomers been fortunate enough to catch brief glimpses of the Universe through lenses fashioned by Nature herself. Background galaxies lensed by foreground galaxy clusters, for example, include some of the most distant brief glimpses of the Universe through galaxies known, and are observed easily only with the aid of the cluslenses fashioned by Nature herself ter-induced magnification (see next section).

Closer to home, the sharp differential magnification generated by binary-star caustics is being used to resolve the surfaces of the background stars. To date, the limb-darkening profiles of four stars - three giants near the centre of the Milky Way and one dwarf star at $\sim 60 \mathrm{pc}$ in the Small Magellanic Cloud (SMC) - have

(a)

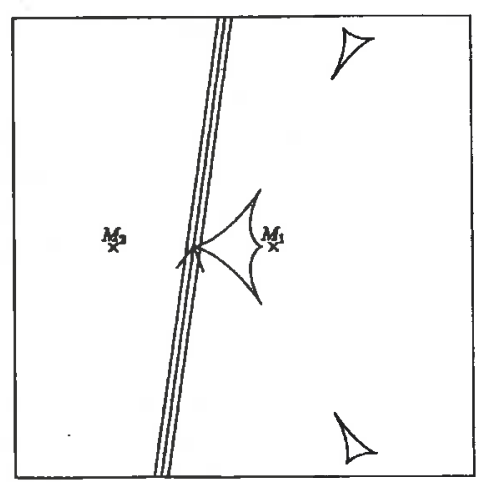

(b)

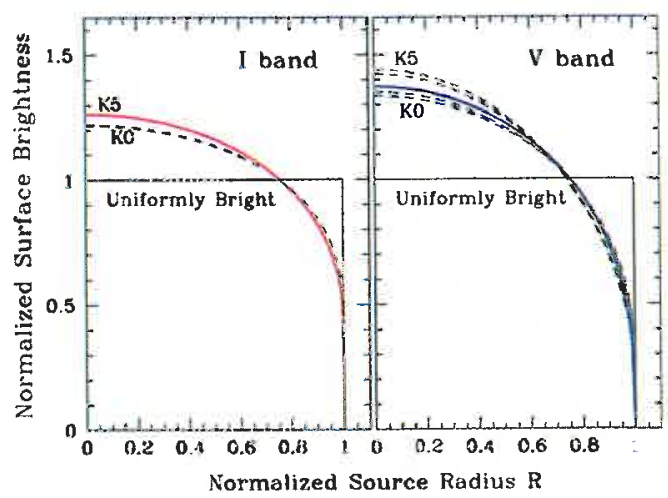

Fig. 2(a): The trajectory of a star (paths of centre and limbs are indicated by the parallel lines) crosses a caustic generated by a foreground binary stellar lens of masses $M_{1}$ and $M_{2}$.

Fig. 2(b): Measurements every 5 minutes of the resulting rapid rise and decline in brightness of the K-giant source enabled deconvolution of the magnification pattern from the star's surface brightness profile. The results at both wavelengths (red and blue) compared well to theory (dashed). (Adapted from Albrow et al.1999, Astrophys.J.522, 1011.) 
been measured. Such measurements are precluded by other techniques due to the miniscule angular sizes (radii of $10^{-7}$ arc seconds at the SMC), and are important since they provide direct tests of theories of stellar atmospheres (Fig. 2). Last year, the high magnification and spatial resolution provided by moving caustics combined with very large telescopes and efficient spectrographs yielded detailed spatially-resolved, spectral information for a giant star in the Galactic Bulge. Qualitatively, the strength of the absorption lines across the face of the giant appears to agree with stellar models; detailed analysis is underway.

\section{Dark and Luminous Lenses of All Sizes}

Much of the excitement and potential of this field lies in the lenses themselves. Barring modification of the law of gravitation on cosmic scales, the mass density of the Universe is dominated by unseen, apparently non-luminous, (dark) matter. Lensing, which is sensitive to mass rather than radiation, is thus an unique and welcome probe.

\section{Galaxy Clusters}

Based on hydrodynamic studies of their hot X-ray gas and virial motion estimates of their constituent galaxies, rich galaxy clusters were inferred to have masses $\sim 10^{15}$ times the mass of the Sun $\left(\mathrm{M}_{\odot}\right)$. Observations of lensing by clusters has now confirmed these estimates directly (to within a factor of two in individual cases), and also allowed detailed mapping of the mass distribution.

Near the cluster centre, where mass densities exceed the critical value required for strong lensing, multiple images of background galaxies are formed (Fig. 3). The position, size, and shape of these giant arcs, especially when they can be associated with their counterpart images, provide information on the asphericity and radial profile of the total cluster mass. In particular, lensing has revealed mass densities that are much more centrally concentrated than previously thought.

Single, small, slightly distorted images are produced by weaker lensing effects in the cluster outskirts. Although individually less dramatic, the statistical orientation of hundreds of these arclets allows mapping of the cluster mass out to several hundred kilo parsecs. Significant substructure is often detected, indicating that, even at the current epoch, many clusters are not dynamically relaxed. Earlier this year, the discovery for the first time of a cluster via weak lensing (later confirmed with optical measurements) demonstrated the usefulness of lensing for measuring density contrasts even in seemingly empty or smooth patches of sky.

\section{Individual Galaxies}

Isolated galaxies also serve as lenses for background sources, but since they are a factor of 100 or more less massive than clusters, the image separations they generate are $\sim 10$ times smaller. Multiple images (like those of quasars discussed above) are only formed if the trajectory passes through the very densest centres of galaxies. The detection of weak lensing by individual galaxies (by co-adding the relative orientations of large statistical samples of foreground-background galaxy pairs) has demonstrated that the typical diffuse halo of dark matter around isolated galaxies contains about $10^{12} \mathrm{M}_{\infty}$ confirming and extending to larger galactocentric radii results derived from the kinematics of gas and stars in the galaxies themselves. The luminous matter associated with galaxies contributes only $3 \%-30 \%$ of this total.

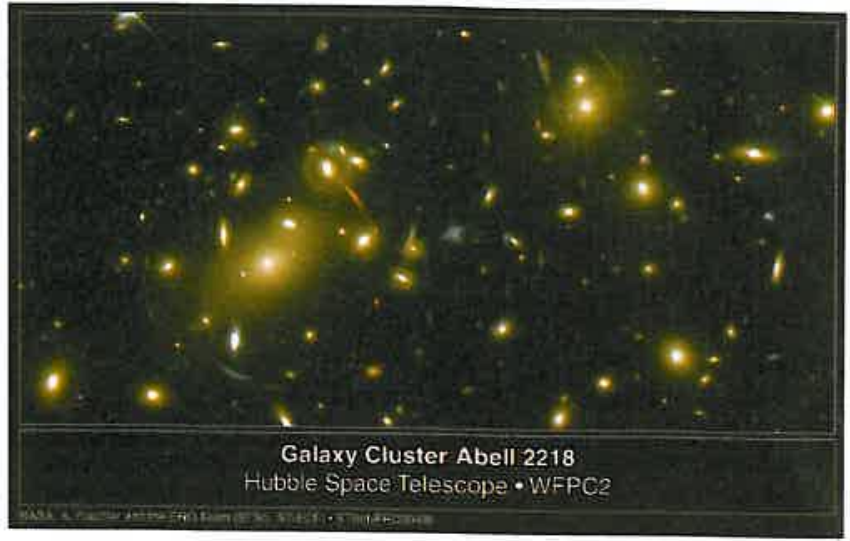

Fig. 3: The cluster Abell 2218 dramatically illustrates the giant arc images of distant background galaxies lensed by the total mass associated with a foreground cluster and its constituent galaxies. (Hubble Space Telescope Archives)

\section{Red, White and Brown Dwarf Stars}

So what is the dark matter? Lensing has not provided a definite answer to this question, but has narrowed the list of candidates, at least in our own galaxy, the Milky Way. If compact objects with stellar or planetary masses comprised the dark matter, their motion in the Galactic potential would occasionally bring them across the line of sight to more distant stars, in particular those of the Large Magellanic Cloud (LMC), a small neighbouring galaxy. At any given time, one LMC star in every million would be "microlensed" by such small, moving dark lenses. Separated by about a milli-arc-second, individual images could not be resolved during the lensing event, but a smooth increase and decline would be observed in the total brightness of the background star. The peak amplitude of the variability is set by the impact parameter $u \equiv \theta_{S} / \theta_{E}$. The duration depends on $\theta_{E}$ and the relative proper motion of lens and source, and for the Milky Way is typically weeks to months.

A decade ago, massive campaigns began searching for these rare microlensing events in the direction of the Galactic centre and LMC. The first events were announced in 1993. Hundreds followed, the majority toward the Milky Way centre. The measured microlensing probability through our stellar disk toward its centre is about that expected from known stellar populations. Outwards through our dark halo toward the LMC, however, the measured value appears to fall short by a factor five from that expected for a halo filled with unseen low-mass red dwarfs, white dwarf stellar remnants, or sub-stellar brown dwarfs. New microlensing surveys are now underway to perform similar experiments through the halo of the large spiral galaxy Andromeda, while very distant (macro)lensed radio sources are being examined for the "twinkling" that would betray small dark microlenses in the halos of the lenses.

\section{Extra-solar Planets}

Extra-solar planets bound to distant stars in the Milky Way would generate small, but extended caustics in the lensing magnification pattern. If probed by a background star, these caustics would cause short-lived (hours to days), but often dramatic, anomalies atop otherwise smooth microlensing light curves. Such planetary signatures are expected to be reasonably frequent 
for Jupiter-mass planets $\left(10^{-3} \mathrm{M}_{\odot}\right)$ in orbits similar to those of Mars or Jupiter. Nevertheless, five years of intense monitoring of Galactic microlensing events has yielded no convincing planetary signals. Analysis of this null result published this year implies that no more than one-third of stellar lenses have Jovianmass planets orbiting in the zone between 1.5 and $4.0 \mathrm{AU}^{1}$ from their parent stars. This limit is consistent with the $\sim 60$ planets orbiting solar-type stars discovered by the Doppler technique, most of which orbit within $1.5 \mathrm{AU}$ where that method is most sensitive.

\section{What's Next?}

What can be expected from lensing studies in the coming decade? Caustics generated by micro-lens networks in distant galaxies may be used to resolve and measure the surface brightness profiles of active galactic nuclei - believed to be associated with massive black holes. Weak lensing measurements over vast portions of the sky may map the large-scale structure of matter (luminous and dark) in the Universe. Advances in observational technique and resource may allow the search for microlensing planetary systems to be pushed into the regime of Earth-mass planets. For a more thorough discussion of these possibilities, and details and references for the scientific work discussed here that were not possible to include in this summary, the reader is referred to the review articles in the bibliography.

\section{Bibliography}

Brainerd, T.G. \& Kochanek, C.S., Gravitational Lensing: Recent Progress and Future Goals, 2001, Astron. Soc. Pacific, Conf. Series 237,

Bartelmann, M. \& Schneider, P., Weak Gravitational Lensing, 2001, Physics Reports 340, 291,

http://www.elsevier.nl/gej-ng/10/36/26/174/30/26/article.pdf

Menzies, J.W. \& Sackett, P.D., Microlensing 2000: A New Era of Microlensing Astrophysics, 2001, Astron. Soc. Pacific, Conf. Series 239

Narayan, P. \& Bartelmann, M., Gravitational Lensing, 1999, in Formation of Structure in the Universe, eds. A. Dekel and J.P. Ostriker (CUP), 360

http://www.mpa-garching.mpg.de/Lenses/Preprints/JeruLect.html

Paczynski, B., Gravitational Microlensing in the Local Group, 1996, Ann.

Rev. Astron. Astrophys. 34, 419

http://astro.AnnualReviews.org/cgi/content/full/34/1/419

Wambsganss, J., Gravitational Lensing in Astronomy, 1998, Living Reviews in Relativity 1, 12

http://www.livingreviews.org/Articles/Volume1/1998-12wamb

\section{About the author}

Penny D. Sackett is Professor of (Extra)Galactic Astronomy at Kapteyn Astronomical Institute in Groningen, The Netherlands. She took her $\mathrm{PhD}$ in physics in 1984 from the University of Pittsburgh in the USA, writing a dissertation on lattice gauge theory. She has served as a Program Director in the Division of Astronomical Sciences at the US National Science Foundation, and was awarded the J. Seward Johnson Fellowship at the Institute for Advanced Study in Princeton, where her interest in microlensing began. She currently serves as a founder and the principal spokesperson for PLANET (Probing Lensing Anomalies NETwork), an international collaboration dedicated to the detailed study of microlenses and their sources in the Milky Way. 